\title{
Thermodynamical Cross-Relations for Superconductors at the Critical Point
}

\author{
B.Grabiec and M.Matlak* \\ Institute of Physics, Silesian University, \\ 4 Uniwersytecka, PL-40-007 Katowice, Poland
}

November 8, 2018

\begin{abstract}
We investigate superconducting systems with the use of the phenomenological Landau's theory of second order phase transitions, including into the considerations the critical behaviour of the chemical potential. We derive in this way a variety of new thermodynamical relations at the critical point. Twelve basic relations connect critical jumps of different thermodynamical quantites (specific heat, chemical potential derivatives with respect to temperature, pressure (volume) and number of particles, volume (pressure) derivatives with respect to temperature and pressure (volume)) with the critical temperature or its derivatives with respect to the number of particles or pressure (volume). These relations allow to find plenty of cross-relations between different quantities at the critical point. The derived formulae can practically be used in many cases to find such thermodynamical quantities at the critical point which are extremely difficult to measure under the assumption that the other ones are already known. We additionally perform a test of the two derived relations by using two-band microscopic model, describing superconducting systems. We calculate the specific heat, order parameter and chemical potential as functions of temperature to show that the tested relations are very well fulfilled.

Subject classification: $05.70 . \mathrm{Fh} ; 74.20 . \mathrm{De} ; 74.25 . \mathrm{Bt}$
\end{abstract}

Introduction First observations of the chemical potential critical behaviour at second order phase transitions has been performed for superconductors (see Refs [1-10]). It has been found that at the critical temperature $T_{s}$ (phase transition: superconductor - normal system) the temperature dependence of the chemical potential exhibits a kink (change in slope), manifesting in this way the appearance of the phase transition (cf also Refs [11-23]). For superconducting systems (see Ref. [6]), an interesting relation between the jump of the chemical potential temperature derivative and the jump of the specific heat at the critical

*matlak@server.phys.us.edu.pl 
point has been derived with the use of the phenomenological, Landau's theory of second order phase transitions (cf Refs $[24,25]$ ). It is, however, possible to include in a more complete way the chemical potential critical behaviour into the general scheme of the Landau's theory for superconducting systems to derive new relations between different thermodynamical quantities at the critical point. Starting from the Gibbs potential we derive six basic relations and further six ones are resulting from the Helmholtz potential. There are two relations connecting the critical jump of the specific heat at constant pressure (volume) and the critical temperature. The next two relate the critical jump of the chemical potential temperature derivative and the critical temperature derivative with respect to the number of particles (constant pressure (volume)). Critical jumps of the volume (pressure) derivatives with respect to temperature and pressure (volume) can be connected with the critical temperature derivatives with respect to pressure (volume), resulting in four relations. The next four relations connect critical jumps of the chemical potential derivatives with respect to pressure (volume) and the number of particles with the critical temperature derivatives with respect to the number of particles and pressure (volume). These twelve basic relations can be combined together by forming ratios of different critical jumps. In this way we eliminate an unknown parameter of the Landau's theory (second Landau's parameter) and we connect directly the mentioned ratios with the critical temperature of the system and its derivatives. These relations can be very useful in the practice because they allow to find an unknown critical jump (or unknown critical temperature derivative) when the other quantities are already known from experimental data. One can additionally eliminate the critical temperature derivatives from these relations to obtain six independent cross-relations between different critical jumps and the critical temperature of the system. These formulae can also be practically used to find an uknown critical jump under the assumption that the other critical jumps are already known. We additionally perform a test of two basic relations, desribed above, when independently calculate the second Landau's parameter from these two relations. To this purpose we applied two-band, microscopic model, describing superconducting systems. The numerical calculation of the specific heat jump and the jump of the order parameter at the critical point, as well as, the critical temperature derivative with respect to the number of particles (electrons) reveals almost the same value for the second Landau's parameter when calculate it from these two independent relations. It seems to be very strange that the analytical derivation of the formulae, we have numerically tested, is extremely difficult to do for this particular, microscopic model (we could not perform it) because of its great complexity (to many implicit equations to solve) whereas the phenomenological derivation is not only general (independent from the assumed microscopic model) and straightforward but also true (!).

Phenomenological relations Within the phenomenological theory of second order phase transitions (see Refs $[24,25]$ ) one assumes the existence of the order parameter $x$. For simplicity, we assume that the system can be described by 
only one order parameter 1 . For superconducting systems the order parameter $x=\left|\Delta_{s}\right|^{2}$ where $\Delta_{s}$ is the superconducting order parameter (or its amplitude in the case of anisotropic superconductivity). The order parameter should fulfil a selfconsistent equation of the general form

$$
x=f(T, p, N, x)
$$

where the variables $T, p, N$ denote the absolute temperature, pressure and number of particles of the system. This equation can formally be resolved to give an equation

$$
x=x(T, p, N)
$$

of such a property that $x=0$ for $T \geqslant T_{s}$ where $T_{s}=T_{s}(p, N)$ is the critical temperature of the transition from superconducting to normal phase. One assumes also that the thermodynamic properties of the system can be desribed by the Gibbs potential

$$
Z=Z(T, p, N, x) .
$$

In the vicinity of $T=T_{s}\left(T<T_{s}\right)$ the order parameter $x$ is very small and therefore we can expand the Gibbs potential (3) into the Taylor series (we retain quadratic terms)

$$
Z(T, p, N, x)=Z_{0}(T, p, N)+a(T, p, N) x+\frac{1}{2} b(T, p, N) x^{2}
$$

where $a$ and $b$ are first and second derivatives of $Z$ with respect to $x$ at the point $x=0$. The minimum principle $\left(\frac{\partial Z}{\partial x}=0, \frac{\partial^{2} Z}{\partial x^{2}}>0\right)$, aplied to (4) results in the expression

$$
x=-\frac{a}{b} \quad(b>0) .
$$

To obtain a general form of the phenomenological parameter $a=a(T, p, N)$ in the vicinity of the critical point we can apply again the Taylor expansion with respect to a small quantity $\left(T-T_{s}\right)$. We get

$$
\begin{aligned}
a(T, p, N) & =a\left(T_{s}+\left(T-T_{s}\right), p, N\right) \\
& \approx a\left(T_{s}, p, N\right)+\left(T-T_{s}\right)\left(\frac{\partial a}{\partial T}\right)_{p, N, T=T_{s}} .
\end{aligned}
$$

However, at $T=T_{s}$ the order parameter $x=0$. It means also that $a\left(T_{s}, p, N\right)=$ 0 (see (6) and (5)). Thus, we obtain

$$
a(T, p, N)= \begin{cases}\left(T-T_{s}\right)\left(\frac{\partial a}{\partial T}\right)_{p, N, T=T_{s}} & , T \leqslant T_{s} \\ 0 & , T>T_{s}\end{cases}
$$

\footnotetext{
${ }^{1}$ See also Note added in proof
} 
and

$$
x=x(T, p, N)=\left\{\begin{array}{ll}
-\frac{\left(T-T_{s}\right)}{b}\left(\frac{\partial a}{\partial T}\right)_{p, N, T=T_{s}} & , T \leqslant T_{s} \\
0 & , T>T_{s}
\end{array} .\right.
$$

Eliminating the phenomenological parameter $b$ from (4) by means of (5) we get

$$
Z=Z_{0}+\frac{1}{2} a x
$$

and using the thermodynamic relations

$$
\begin{gathered}
S=-\left(\frac{\partial Z}{\partial T}\right)_{p, N}, \\
S_{0}=-\left(\frac{\partial Z_{0}}{\partial T}\right)_{p, N}
\end{gathered}
$$

we can write

$$
S-S_{0}=-\frac{1}{2}\left(\frac{\partial a}{\partial T}\right)_{p, N} x-\frac{1}{2} a\left(\frac{\partial x}{\partial T}\right)_{p, N} .
$$

The specific heat difference, produced by (12) is given by relation

$$
\begin{aligned}
c_{p, N}(T)-c_{p, N}^{(0)}(T)= & T\left(\frac{\partial S}{\partial T}\right)_{p, N}-T\left(\frac{\partial S_{0}}{\partial T}\right)_{p, N} \\
= & -\frac{T}{2}\left(\frac{\partial^{2} a}{\partial T^{2}}\right)_{p, N} x-T\left(\frac{\partial a}{\partial T}\right)_{p, N}\left(\frac{\partial x}{\partial T}\right)_{p, N} \\
& -\frac{a T}{2}\left(\frac{\partial^{2} x}{\partial T^{2}}\right)_{p, N} .
\end{aligned}
$$

To obtain the jump of the specific heat $\Delta c_{p, N}$ at the critical point we have to insert $T=T_{s}$ into (13) taking also into accout that $x=a=0$ at $T=T_{s}$ (see (7), (8)). Thus, we obtain

$$
\Delta c_{p, N}=-T_{s}\left(\frac{\partial a}{\partial T}\right)_{p, N, T=T_{s}}\left(\frac{\partial x}{\partial T}\right)_{p, N, T=T_{s}} .
$$

However, when looking at (8) we can see that

$$
\left(\frac{\partial x}{\partial T}\right)_{p, N, T=T_{s}}=-\frac{1}{b}\left(\frac{\partial a}{\partial T}\right)_{p, N, T=T_{s}} \equiv \Delta\left(\frac{\partial x}{\partial T}\right)_{p, N, T=T_{s}}
$$

where $\Delta\left(\frac{\partial x}{\partial T}\right)_{p, N, T=T_{s}}$ is just the jump of the temperature derivative of the order parameter at $T \stackrel{s}{=} T_{s}$ because the order parameter $x$ vanishes for $T \geqslant T_{s}$. 
Thus, we can rewrite (14) with the use of (15) by eliminating $\left(\frac{\partial a}{\partial T}\right)_{p, N, T=T_{s}}$. We obtain

$$
\Delta c_{p, N}=b T_{s}\left[\Delta\left(\frac{\partial x}{\partial T}\right)_{p, N, T=T_{s}}\right]^{2} .
$$

The chemical potential of the system can be introduced from the general expression for the Gibbs potential using the relations

$$
\mu=\left(\frac{\partial Z}{\partial N}\right)_{T, p} \quad, \quad \mu_{0}=\left(\frac{\partial Z_{0}}{\partial N}\right)_{T, p} .
$$

These relations applied to (9) result

$$
\mu=\mu_{0}+\frac{1}{2}\left(\frac{\partial a}{\partial N}\right)_{T, p} x+\frac{1}{2} a\left(\frac{\partial x}{\partial N}\right)_{T, p} .
$$

Thus, the jump of the chemical potential temperature derivative at $T=T_{s}$ $\left(x=0, a=0\right.$ for $\left.T \geqslant T_{s}\right)$ is equal to

$$
\begin{aligned}
\Delta\left(\frac{\partial \mu}{\partial T}\right)_{p, N, T=T_{s}} \equiv & \left(\frac{\partial \mu}{\partial T}\right)_{p, N, T=T_{s}}-\left(\frac{\partial \mu_{0}}{\partial T}\right)_{p, N, T=T_{s}} \\
= & \frac{1}{2}\left(\frac{\partial a}{\partial N}\right)_{T=T_{s}, p}\left(\frac{\partial x}{\partial T}\right)_{p, N, T=T_{s}} \\
& +\frac{1}{2}\left(\frac{\partial a}{\partial T}\right)_{p, N, T=T_{s}}\left(\frac{\partial x}{\partial N}\right)_{T=T_{s}, p} .
\end{aligned}
$$

Using (7), (8) and (15) we find $\left(T_{s}=T_{s}(p, N)\right)$ :

$$
\begin{aligned}
\left(\frac{\partial a}{\partial N}\right)_{T=T_{s}, p} & =-\left(\frac{\partial T_{s}}{\partial N}\right)_{p}\left(\frac{\partial a}{\partial T}\right)_{p, N, T=T_{s}} \\
& =b\left(\frac{\partial T_{s}}{\partial N}\right)_{p}\left[\Delta\left(\frac{\partial x}{\partial T}\right)_{p, N, T=T_{s}}\right]
\end{aligned}
$$

and

$$
\begin{aligned}
\left(\frac{\partial x}{\partial N}\right)_{T=T_{s}, p} & =\frac{1}{b}\left(\frac{\partial T_{s}}{\partial N}\right)_{p}\left(\frac{\partial a}{\partial T}\right)_{p, N, T=T_{s}} \\
& =-\left(\frac{\partial T_{s}}{\partial N}\right)_{p}\left[\Delta\left(\frac{\partial x}{\partial T}\right)_{p, N, T=T_{s}}\right] .
\end{aligned}
$$

Introducing (20) and (21) into (19) we get

$$
\Delta\left(\frac{\partial \mu}{\partial T}\right)_{p, N, T=T_{s}}=b\left(\frac{\partial T_{s}}{\partial N}\right)_{p}\left[\Delta\left(\frac{\partial x}{\partial T}\right)_{p, N, T=T_{s}}\right]^{2} .
$$


It is also easy to find that

$$
\begin{gathered}
\left(\frac{\partial a}{\partial p}\right)_{T=T_{s}, N}=-\left(\frac{\partial T_{s}}{\partial p}\right)_{N}\left(\frac{\partial a}{\partial T}\right)_{p, N, T=T_{s}}=b\left(\frac{\partial T_{s}}{\partial p}\right)_{N} \Delta\left(\frac{\partial x}{\partial T}\right)_{p, N, T=T_{s}}, \\
\left(\frac{\partial x}{\partial p}\right)_{T=T_{s}, N}=\frac{1}{b}\left(\frac{\partial T_{s}}{\partial p}\right)_{N}\left(\frac{\partial a}{\partial T}\right)_{p, N, T=T_{s}}=-\left(\frac{\partial T_{s}}{\partial p}\right)_{N} \Delta\left(\frac{\partial x}{\partial T}\right)_{p, N, T=T_{s}} .
\end{gathered}
$$

We can perform similar calculations as above to find critical jumps of the following thermodynamical quantities: $\Delta\left(\frac{\partial V}{\partial T}\right)_{p, N, T=T_{s}}, \Delta\left(\frac{\partial \mu}{\partial p}\right)_{T=T_{s}, N}, \Delta\left(\frac{\partial \mu}{\partial N}\right)_{T=T_{s}, p}$ and $\Delta\left(\frac{\partial V}{\partial p}\right)_{T=T_{s}, N}$. Starting from the expression for the Gibbs potential (9) with the use of the thermodynamical relations $\mu=\left(\frac{\partial Z}{\partial T}\right)_{T, p}\left(\mu_{0}=\left(\frac{\partial Z_{0}}{\partial T}\right)_{T, p}\right)$, $V=\left(\frac{\partial Z}{\partial p}\right)_{T, N}\left(V_{0}=\left(\frac{\partial Z_{0}}{\partial p}\right)_{T, N}\right)$ and the above formulae (15), (20), (21), (23) and (24) we obtain

$$
\begin{gathered}
\Delta\left(\frac{\partial V}{\partial T}\right)_{p, N, T=T_{s}}=b\left(\frac{\partial T_{s}}{\partial p}\right)_{N}\left[\Delta\left(\frac{\partial x}{\partial T}\right)_{p, N, T=T_{s}}\right]^{2} \\
\Delta\left(\frac{\partial \mu}{\partial p}\right)_{T=T_{s}, N}=-b\left(\frac{\partial T_{s}}{\partial N}\right)_{p}\left(\frac{\partial T_{s}}{\partial p}\right)_{N}\left[\Delta\left(\frac{\partial x}{\partial T}\right)_{p, N, T=T_{s}}\right]^{2} \\
\Delta\left(\frac{\partial \mu}{\partial N}\right)_{T=T_{s}, p}=-b\left[\left(\frac{\partial T_{s}}{\partial N}\right)_{p}\right]^{2}\left[\Delta\left(\frac{\partial x}{\partial T}\right)_{p, N, T=T_{s}}\right]^{2}
\end{gathered}
$$

and

$$
\Delta\left(\frac{\partial V}{\partial p}\right)_{T=T_{s}, N}=-b\left[\left(\frac{\partial T_{s}}{\partial p}\right)_{N}\right]^{2}\left[\Delta\left(\frac{\partial x}{\partial T}\right)_{p, N, T=T_{s}}\right]^{2} .
$$

Using the Maxwell relations $\left(\frac{\partial S}{\partial p}\right)_{T, N}=-\left(\frac{\partial V}{\partial T}\right)_{p, N},\left(\frac{\partial S}{\partial N}\right)_{T, p}=-\left(\frac{\partial \mu}{\partial T}\right)_{p, N}$ and $\left(\frac{\partial V}{\partial N}\right)_{T, p}=\left(\frac{\partial \mu}{\partial p}\right)_{T, N}$ we can see that the other critical jumps $\Delta\left(\frac{\partial S}{\partial p}\right)_{T=T_{s}, N}$ , $\Delta\left(\frac{\partial S}{\partial N}\right)_{T=T_{s}, p}$ and $\Delta\left(\frac{\partial V}{\partial N}\right)_{T=T_{s}, p}$ can easily be obtained from (25), (22) and (26), respectively. In other words, there are only six independent expressions describing the critical jumps of the first derivatives ((16), (22) and (25)-(28)). These expressions are very important because with the use of them we can find many interesting cross-relations. Dividing e.g. (22) and (25)-(28) by (16) 
we obtain five independent ratios (the specific heat critical jump is finite for superconductors):

$$
\begin{gathered}
\frac{\Delta\left(\frac{\partial \mu}{\partial T}\right)_{p, N, T=T_{s}}}{\Delta c_{p, N}}=\left(\frac{\partial \ln T_{s}}{\partial N}\right)_{p} \\
\frac{\Delta\left(\frac{\partial V}{\partial T}\right)_{p, N, T=T_{s}}}{\Delta c_{p, N}}=\left(\frac{\partial \ln T_{s}}{\partial p}\right)_{N}, \\
\frac{\Delta\left(\frac{\partial \mu}{\partial p}\right)_{T=T_{s}, N}}{\Delta c_{p, N}}=-\left(\frac{\partial \ln T_{s}}{\partial N}\right)_{p}\left(\frac{\partial T_{s}}{\partial p}\right)_{N}, \\
\frac{\Delta\left(\frac{\partial \mu}{\partial N}\right)_{T=T_{s}, p}}{\Delta c_{p, N}}=-\left(\frac{\partial \ln T_{s}}{\partial N}\right)_{p}\left(\frac{\partial T_{s}}{\partial N}\right)_{p},
\end{gathered}
$$

and

$$
\frac{\Delta\left(\frac{\partial V}{\partial p}\right)_{T=T_{s}, N}}{\Delta c_{p, N}}=-\left(\frac{\partial \ln T_{s}}{\partial p}\right)_{N}\left(\frac{\partial T_{s}}{\partial p}\right)_{N} .
$$

It is, however, possible to find also another ratios (equivalent to the above relations). When eliminate the derivatives $\left(\frac{\partial T_{s}}{\partial N}\right)_{p}$ and $\left(\frac{\partial T_{s}}{\partial p}\right)_{N}$ from the above expressions we can obtain three independent cross-relations connecting different critical jumps:

$$
\begin{gathered}
\Delta\left(\frac{\partial \mu}{\partial p}\right)_{T=T_{s}, N} \cdot \Delta c_{p, N}=-T_{s} \Delta\left(\frac{\partial \mu}{\partial T}\right)_{p, N, T=T_{s}} \cdot \Delta\left(\frac{\partial V}{\partial T}\right)_{p, N, T=T_{s}} \\
\Delta\left(\frac{\partial \mu}{\partial N}\right)_{T=T_{s}, p} \cdot \Delta c_{p, N}=-T_{s}\left[\Delta\left(\frac{\partial \mu}{\partial T}\right)_{p, N, T=T_{s}}\right]^{2}
\end{gathered}
$$

and

$$
\Delta\left(\frac{\partial V}{\partial p}\right)_{T=T_{s}, N} \cdot \Delta c_{p, N}=-T_{s}\left[\Delta\left(\frac{\partial V}{\partial T}\right)_{p, N, T=T_{s}}\right]^{2} .
$$

These relations allow to find some critical jumps which are difficult to measure, knowing the other from experiments, without necessity to know the derivatives of the critical temperature.

We can also derive several additional relations, when using the thermodynamical relationship resulting from the Gibbs potential (1)

$$
V=\left(\frac{\partial Z}{\partial p}\right)_{T, N}=V(T, p, N, x) .
$$

The equation (37) can formally be resolved to obtain the equation of state

$$
p=p(T, V, N, x) .
$$


We can insert it into (1) to obtain

$$
\bar{Z}=\bar{Z}(T, V, N, x)
$$

which is nothing else but the Helmholtz potential. We can further apply exactly the same procedure as described above starting again with the Taylor expansion of the expression (39) with respect to the order parameter in the vicinity of the critical point. Therefore there is no need to demonstrate here all the details of the performed calculations. We restrict ourselves to present only final results. The analogous formulae (cf (16), (22) and (25)-(28)) for the critical jumps, resulting from (39), have the form

$$
\begin{gathered}
\Delta c_{V, N}=b T_{s}\left[\Delta\left(\frac{\partial x}{\partial T}\right)_{V, N, T=T_{s}}\right]^{2}, \\
\Delta\left(\frac{\partial \mu}{\partial T}\right)_{V, N, T=T_{s}}=b\left(\frac{\partial T_{s}}{\partial N}\right)_{V}\left[\Delta\left(\frac{\partial x}{\partial T}\right)_{V, N, T=T_{s}}\right]^{2} \\
\Delta\left(\frac{\partial p}{\partial T}\right)_{V, N, T=T_{s}}=-b\left(\frac{\partial T_{s}}{\partial V}\right)_{N}\left[\Delta\left(\frac{\partial x}{\partial T}\right)_{V, N, T=T_{s}}\right]^{2} \\
\Delta\left(\frac{\partial \mu}{\partial V}\right)_{T=T_{s}, N}=-b\left(\frac{\partial T_{s}}{\partial N}\right)_{V}\left(\frac{\partial T_{s}}{\partial V}\right)_{N}\left[\Delta\left(\frac{\partial x}{\partial T}\right)_{V, N, T=T_{s}}\right]^{2} \\
\Delta\left(\frac{\partial \mu}{\partial N}\right)_{T=T_{s}, V}=-b\left[\left(\frac{\partial T_{s}}{\partial N}\right)_{V}\right]^{2}\left[\Delta\left(\frac{\partial x}{\partial T}\right)_{V, N, T=T_{s}}\right.
\end{gathered}
$$

and

$$
\Delta\left(\frac{\partial p}{\partial V}\right)_{T=T_{s}, N}=b\left[\left(\frac{\partial T_{s}}{\partial V}\right)_{N}\right]^{2}\left[\Delta\left(\frac{\partial x}{\partial T}\right)_{V, N, T=T_{s}}\right]^{2} .
$$

The constant $b$ has the same meaning as before but actually $b=b\left(T_{s}, V, N\right)$. To derive (40)-(45) we have applied thermodynamical relations $S=-\left(\frac{\partial \bar{Z}}{\partial T}\right)_{V, N}$, $p=-\left(\frac{\partial \bar{Z}}{\partial V}\right)_{T, N}$ and $\mu=\left(\frac{\partial \bar{Z}}{\partial N}\right)_{T, V}\left(S_{0}=-\left(\frac{\partial \bar{Z}_{0}}{\partial T}\right)_{V, N}, p_{0}=-\left(\frac{\partial \bar{Z}_{0}}{\partial V}\right)_{T, N}\right.$ and $\mu_{0}=\left(\frac{\partial \bar{Z}_{0}}{\partial N}\right)_{T, V}$ where $\bar{Z}_{0}=\bar{Z}_{0}(T, V, N)$ in analogy to (4)). Because of the Maxwell relations $\left(\frac{\partial S}{\partial V}\right)_{T, N}=\left(\frac{\partial p}{\partial T}\right)_{V, N},\left(\frac{\partial p}{\partial N}\right)_{T, V}=-\left(\frac{\partial \mu}{\partial V}\right)_{T, N}$ and $\left(\frac{\partial S}{\partial N}\right)_{T, V}=-\left(\frac{\partial \mu}{\partial T}\right)_{V, N}$ the critical jumps $\Delta\left(\frac{\partial S}{\partial V}\right)_{T=T_{s}, N}, \Delta\left(\frac{\partial p}{\partial N}\right)_{T=T_{s}, V}$ and 
$\Delta\left(\frac{\partial S}{\partial N}\right)_{T=T_{s}, V}$ can easily be found from (42), (43) and (41), respectively, therefore there are again only six independent formulae (40)-(45). When e.g. devide (41)-(45) by (40) we get:

$$
\begin{gathered}
\frac{\Delta\left(\frac{\partial \mu}{\partial T}\right)_{V, N, T=T_{s}}}{\Delta c_{V, N}}=\left(\frac{\partial \ln T_{s}}{\partial N}\right)_{V}, \\
\frac{\Delta\left(\frac{\partial p}{\partial T}\right)_{V, N, T=T_{s}}}{\Delta c_{V, N}}=-\left(\frac{\partial \ln T_{s}}{\partial V}\right)_{N}, \\
\frac{\Delta\left(\frac{\partial \mu}{\partial V}\right)_{T=T_{s}, N}}{\Delta c_{V, N}}=-\left(\frac{\partial \ln T_{s}}{\partial N}\right)_{V}\left(\frac{\partial T_{s}}{\partial V}\right)_{N}, \\
\frac{\Delta\left(\frac{\partial \mu}{\partial N}\right)_{T=T_{s}, V}}{\Delta c_{V, N}}=-\left(\frac{\partial \ln T_{s}}{\partial N}\right)_{V}\left(\frac{\partial T_{s}}{\partial N}\right)_{V}
\end{gathered}
$$

and

$$
\frac{\Delta\left(\frac{\partial p}{\partial V}\right)_{T=T_{s}, N}}{\Delta c_{V, N}}=\left(\frac{\partial \ln T_{s}}{\partial V}\right)_{N}\left(\frac{\partial T_{s}}{\partial V}\right)_{N} .
$$

There are, however, many other possilities to form the ratios, leading to equivalent relations.

At last, when eliminate from (46)-(50) the critical temperature derivatives, we obtain (cf (34)-(36))

$$
\begin{gathered}
\Delta\left(\frac{\partial \mu}{\partial V}\right)_{T=T_{s}, N} \cdot \Delta c_{V, N}=T_{s} \Delta\left(\frac{\partial \mu}{\partial T}\right)_{V, N, T=T_{s}} \cdot \Delta\left(\frac{\partial p}{\partial T}\right)_{V, N, T=T_{s}} \\
\Delta\left(\frac{\partial \mu}{\partial N}\right)_{T=T_{s}, V} \cdot \Delta c_{V, N}=-T_{s}\left[\Delta\left(\frac{\partial \mu}{\partial T}\right)_{V, N, T=T_{s}}\right]^{2}
\end{gathered}
$$

and

$$
\Delta\left(\frac{\partial p}{\partial V}\right)_{T=T_{s}, N} \cdot \Delta c_{V, N}=T_{s}\left[\Delta\left(\frac{\partial p}{\partial T}\right)_{V, N, T=T_{s}}\right]^{2} .
$$

Thus, the derived formulae (16), (22), (25)-(28) together with (40)-(45) allowed to find many useful thermodynamical cross-relations for superconducting systems at the critical point. 
Numerical Test: superconductor - normal system transition In the following we perform a validity test of the formulae (40) and (41) when using microscopic model, describing superconducting properties of a system with two hybrydized electronic bands. The Cooper - pairs attraction, responsible for the superconducting properties, we choose in the form of the intersite interaction restricted to only one band (one superconducting order parameter). The Hamiltonian of this model can be written in the form (great canonical ensemble)

$$
\bar{H}=H-\mu N
$$

where

$$
\begin{aligned}
H= & \sum_{\alpha=1}^{2} \sum_{i, j, \sigma} t_{i, j}^{(\alpha)} c_{i, \sigma}^{(\alpha)^{+}} c_{i, \sigma}^{(\alpha)}+V \sum_{i, \sigma}\left(c_{i, \sigma}^{(1)^{+}} c_{i, \sigma}^{(2)}+c_{i, \sigma}^{(2)^{+}} c_{i, \sigma}^{(1)}\right) \\
& +\sum_{i, j} R_{i, j} c_{i, \uparrow}^{(2)^{+}} c_{j, \downarrow}^{(2)^{+}} c_{j, \downarrow}^{(2)} c_{i, \uparrow}^{(2)}
\end{aligned}
$$

and

$$
N=\sum_{\alpha=1}^{2} \sum_{i, \sigma} n_{i, \sigma}^{(\alpha)} .
$$

Two electronic bands are characterized by the hopping intergrals $t_{i, j}^{(\alpha)}(\alpha=1,2$; $\left.t_{i, i}^{(1)}=0, t_{i, i}^{(2)}=t\right)$. The parameters $V$ and $R_{i, j}$ denote the hybridization and intersite Cooper - pairs attraction, respectively. The electronic annihilation (creation) operators $(\alpha=1,2 ; \sigma=\uparrow, \downarrow)$ are denoted by $c_{i, \sigma}^{(\alpha)}\left(c_{i, \sigma}^{(\alpha)^{+}}\right)$, respectively, where $i$ is the lattice site index. For simplicity, we consider simple cubic (sc) lattice. Similarly to Ref. [26] we apply the mean field approximation (see the third term in (55))

$$
\begin{aligned}
c_{i, \uparrow}^{(2)^{+}} c_{j, \downarrow}^{(2)^{+}} c_{j, \downarrow}^{(2)} c_{i, \uparrow}^{(2)} \approx & \left\langle c_{i, \uparrow}^{(2)^{+}} c_{j, \downarrow}^{(2)^{+}}\right\rangle c_{j, \downarrow}^{(2)} c_{i, \uparrow}^{(2)}+\left\langle c_{j, \downarrow}^{(2)} c_{i, \uparrow}^{(2)}\right\rangle c_{i, \uparrow}^{(2)^{+}} c_{j, \downarrow}^{(2)^{+}} \\
& -\left\langle c_{i, \uparrow}^{(2)^{+}} c_{j, \downarrow}^{(2)^{+}}\right\rangle\left\langle c_{j, \downarrow}^{(2)} c_{i, \uparrow}^{(2)}\right\rangle .
\end{aligned}
$$

Introducing (57) into (55) and applying Fourier transformation we obtain

$$
\begin{aligned}
H= & -\sum_{\mathbf{k}} \Delta_{\mathbf{k}}^{*}\left\langle c_{-\mathbf{k} \downarrow}^{(2)} c_{\mathbf{k} \uparrow}^{(2)}\right\rangle+\sum_{\alpha=1}^{2} \sum_{\mathbf{k}} \varepsilon_{\mathbf{k}}^{(\alpha)}\left(n_{\mathbf{k}, \uparrow}^{(\alpha)}+n_{-\mathbf{k}, \downarrow}^{(\alpha)}\right) \\
& +V \sum_{\mathbf{k}}\left(c_{\mathbf{k}, \uparrow}^{(1)^{+}} c_{\mathbf{k}, \uparrow}^{(2)}+c_{\mathbf{k}, \uparrow}^{(2)^{+}} c_{\mathbf{k}, \uparrow}^{(1)}+c_{-\mathbf{k}, \downarrow}^{(1)^{+}} c_{-\mathbf{k}, \downarrow}^{(2)}+c_{-\mathbf{k}, \downarrow}^{(2)^{+}} c_{-\mathbf{k}, \downarrow}^{(1)}\right) \\
& +\sum_{\mathbf{k}} \Delta_{\mathbf{k}}^{*} c_{-\mathbf{k}, \downarrow}^{(2)} c_{\mathbf{k}, \uparrow}^{(2)}+\sum_{\mathbf{k}} \Delta_{\mathbf{k}} c_{\mathbf{k}, \uparrow}^{(2)} c_{-\mathbf{k}, \downarrow}^{(2)},
\end{aligned}
$$




$$
N=\sum_{\alpha=1}^{2} \sum_{\mathbf{k}}\left(n_{\mathbf{k}, \uparrow}^{(\alpha)}+n_{-\mathbf{k}, \downarrow}^{(\alpha)}\right)
$$

where

$$
\begin{gathered}
\Delta_{\mathbf{k}}=\frac{1}{\mathcal{N}} \sum_{\mathbf{k}} R\left(\mathbf{k}-\mathbf{k}^{\prime}\right)\left\langle\mathbf{c}_{-\mathbf{k}, \downarrow}^{(\mathbf{2})} \mathbf{c}_{\mathbf{k}, \uparrow}^{(\mathbf{2})}\right\rangle, \\
\varepsilon_{\mathbf{k}}^{(1)}=-\frac{W^{(1)}}{6} F(\mathbf{k}), \\
\varepsilon_{\mathbf{k}}^{(2)}=t-\frac{W^{(2)}}{6} F(\mathbf{k}), \\
R(\mathbf{k})=2 R_{0} F(\mathbf{k}), \\
F(\mathbf{k})=\cos \left(k_{\mathrm{x}} a\right)+\cos \left(k_{\mathrm{y}} a\right)+\cos \left(k_{\mathrm{z}} a\right)
\end{gathered}
$$

and $\mathcal{N}$ is the number of lattice points. We assume that the bandwidth $W^{(2)}=W$ and $W^{(1)}=\delta W(\delta \ll 1)$. In the following we assume that (cf Ref. [26])

$$
\Delta_{\mathbf{k}}=\Delta_{s} F(\mathbf{k})
$$

where $\Delta_{s}$ (we assume $\Delta_{s}^{*}=\Delta_{s}$ ) is the amplitude of the superconducting order parameter ( $s^{*}$ - symmetry). Looking at the expressions (61)-(63) and (65) we see that the only dispression in our system is generated by the formfactor (64) of the sc lattice. It means that we can convert $\frac{1}{\mathcal{N}} \sum_{\mathbf{k}}(\ldots) \rightarrow \int_{-\frac{W}{2}}^{\frac{W}{2}} \rho_{0}(\varepsilon)(\ldots) d \varepsilon$ where $\rho_{0}(\varepsilon)$ is the true density of states of the sc lattice given by the Jelitto formula [27]. Applying the standard Green's function approach (cf e.g. [28]) we can find a set of transcendental equations of the type $(\alpha=1,2 ; \sigma=\uparrow, \downarrow)$

$$
\begin{gathered}
\left\langle n_{\sigma}^{(\alpha)}\right\rangle=f_{\sigma}^{(\alpha)}\left(T, \Delta_{s}^{2}, \mu\right), \\
\Delta_{s}^{2}=\Delta_{s}^{2} f\left(T, \Delta_{s}^{2}, \mu\right)
\end{gathered}
$$

and

$$
\sum_{\alpha=1}^{2} \sum_{\sigma}\left\langle n_{\sigma}^{(\alpha)}\right\rangle=n
$$

where $n=\frac{\langle N\rangle}{\mathcal{N}}$ is the average number of electrons per lattice site. The calculations of all necessary quantities to check the formulae (40) and (41) are very laborious and lead to very complicated expressions (to long to present here). Therefore we are forced to describe only the general calculation scheme and 

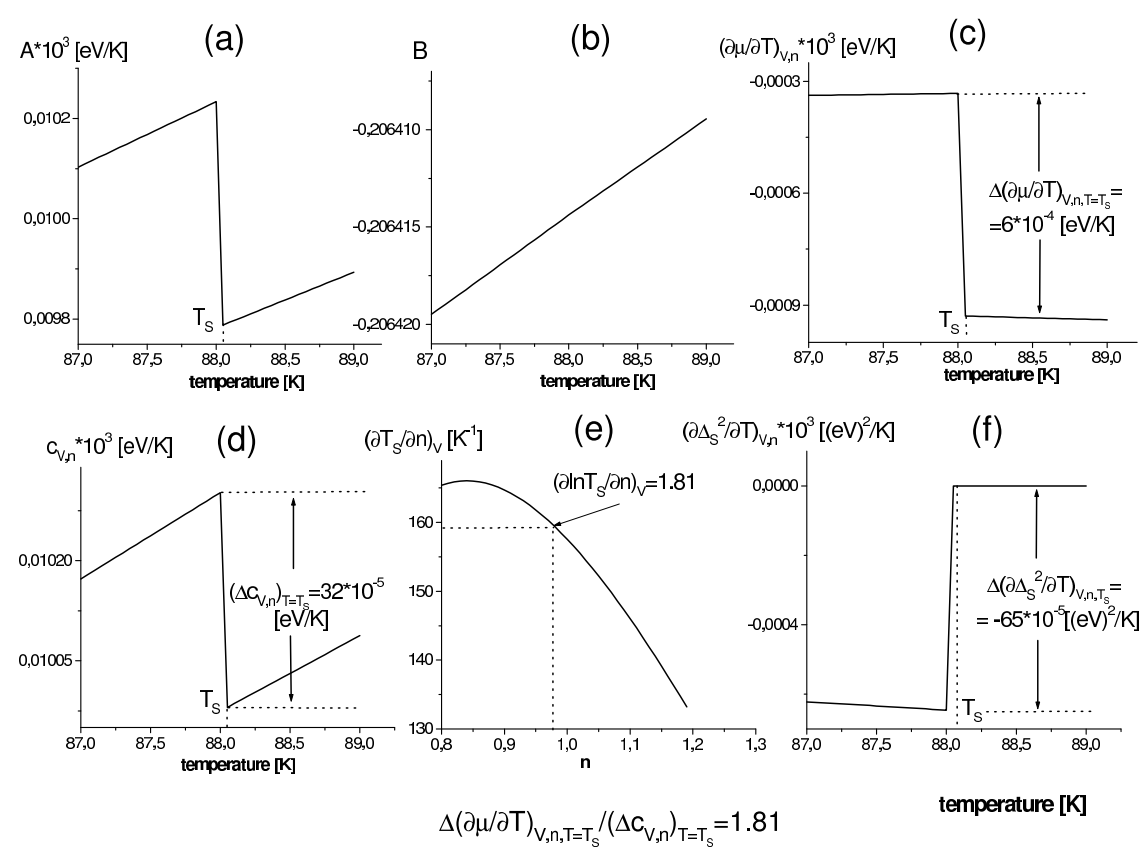

Figure 1: Plot of the coefficients $A$ (Fig. 1a), $B$ (Fig. 1b) and $\left(\frac{\partial \mu}{\partial T}\right)_{V, n}$ (Fig. 1c) entering into the specific heat per lattice site (Fig. 1d) as functions of temperature in the vicinity of the critical point. The plots in Fig. 1e, f show the superconducting critical temperature derivative with respect to the average number of electrons per lattice site $n$ as function of $n$ and the temperature derivative of the order parameter $\Delta_{s}^{2}$ as function of temperature. The model parameters: $W=3 \mathrm{eV}, \delta=0.25, R_{0}=0.6 \mathrm{eV}, t=1 \mathrm{eV}, V=0.15 \mathrm{eV}, n=0.97$.

to present final, numerical results. The implicit equations (66)-(68) allow to calculate the temperature dependence of the square amplitude $\Delta_{s}^{2}$ (it is just the order parameter in the sense of the Landau's theory) and $\mu$ as function of temperature. The calculation of the specific heat per lattice site $c_{V, n}$ can be performed with the use of (58) when calculating

$$
\widetilde{\langle H\rangle}=\frac{\langle H\rangle}{\mathcal{N}}
$$

and differentiating it with respect to $T$. This differentiation is, however, very laborious because $\Delta_{s}^{2}$ and $\mu$ fulfil the equations (66)-(68) and are also temperature dependent. In other words, we have to differentiate them with respect to $T$ to calculate the temeprature derivatives of $\Delta_{s}^{2}$ and $\mu$ (necessary to check the formulae (40) and (41). The transition temperature $T_{s}$ (phase transition from superconducting to normal state) can be obtained from the equation (67). It is easy to see that $T_{s}$ should fulfil the following equation

$$
f\left(T_{s}, \mu\right)=1 .
$$


The function $f$ in (70) is exactly the same as in the rhs of (67) but we have additionally assumed that the order parameter is equal to zero $\left(T=T_{s}\right)$. We should also calculate the derivative $\left(\frac{\partial T_{s}}{\partial n}\right)_{V}$. It can be performed by the differentiation of the equations (70), (66) and (68) with respect to $n$ (we put $T=T_{s}$ therein (the order parameter is equal to zero)). In such a way we have all necessary quantities to check the formulae (40) and (41). These formulae we should first rewrite in the following way $\left(N \longrightarrow\langle N\rangle=\mathcal{N} n, x=\Delta_{s}^{2}\right.$, $b \longrightarrow \bar{b}=\frac{b}{\mathcal{N}}$ (the second Landau's parameter per lattice site)). Thus, we obtain

$$
\frac{\Delta c_{V, n}}{T_{s}\left[\Delta\left(\frac{\partial \Delta_{s}^{2}}{\partial T}\right)_{V, n, T=T_{s}}\right]^{2}}=\bar{b}
$$

and

$$
\frac{\Delta\left(\frac{\partial \mu}{\partial T}\right)_{V, n, T=T_{s}}}{\left(\frac{\partial T_{s}}{\partial n}\right)_{V}\left[\Delta\left(\frac{\partial \Delta_{s}^{2}}{\partial T}\right)_{V, n, T=T_{s}}\right]^{2}}=\bar{b} .
$$

The specific heat per lattice site can be written in the form

$$
c_{V, n}(T)=A(T)+B(T)\left(\frac{\partial \mu}{\partial T}\right)_{V, n} .
$$

The results of our calculations are presented in Fig. 1. We see (Fig. 1a, c) that $A(T)$ and the temperature derivative of the chemical potential exhibit jumps at $T=T_{s}$ whereas the coefficient $B(T)$ is a continuous function at the critical point (Fig. 1b). It results in the jump of the specific heat per lattice site $c_{V, n}$ (Fig. 1d) at $T=T_{s}$. It would be interseting to know whether the temperature behaviour of the coefficients (Fig.1a, b, c) in (73) is a general property of all superconductors (when write the specific heat in the form (73)) or only a property of this particular model. This question needs a verification from the experimental (nobody has proved it till now) and also from the theoretical point of view (when consider another microscopic models). From the other hand, the model we have applied here, should qualitatively reproduce all general properties of superconductors. Therefore it is very probably that only coefficient $A$ and the temperature derivative of the chemical potential exibit jumps at the critical point whereas the coefficient $B$ is a continuous function for these materials. The plots of the derivatives $\left(\frac{\partial T_{s}}{\partial n}\right)_{V}$ and $\left(\frac{\partial \Delta_{s}^{2}}{\partial T}\right)_{V, n}$ are given in Fig. 1e and Fig. 1f, respectively. Calculating the lhs of (71) and (72) independently by means of the data displayed in the Figs $1 \mathrm{c}-\mathrm{f}$ we obtain almost the same value of the second Landau's parameter per lattice site $\bar{b} \approx 8.6 \mathrm{eV}^{-3}$. It also means that the formula (46) is automatically fulfilled (see Fig. 1c, d, e). 
Conclusions The general, phenomenological relations (16), (22) and (25)(28) together with their analogs (40)-(45) seem to be very important for superconducting systems in the practice because they connect different macroscopic thermodynamical quantities at the critical point. They also allowed to obtain interesting cross-relations (29)-(36) and (46)-(53) between different critical jumps and the critical temperature derivatives or between the separate jumps. Due to a variety of relations we have a possibility to estimate in an indirect way many of the critical jumps without performing experiments. Such a possibility can especially be interesting in many cases where experiments are difficult to perform and the information gained in this way can be decisive as e.g. the indication for the hole-type superconductivity in the high- $T_{c}$ superconductor $\mathrm{YBa}_{2} \mathrm{Cu}_{3} \mathrm{O}_{7-\delta}$ (see Refs [6], [7] for details).

Note added in proof The derived critical relations (29)-(36) and (46)-(53) are also valid for a more general case of superconducting, correlated systems with many order parameters (multiband systems). As a consequence of electronic correlations the critical temperature $T_{s}$ is the same for all order parameters (all of them vanish for $\left.T \geq T_{s}\right)$. To make a proof of the statement that the relations (29)-(36) and (46)-(53) are valid in this case we consider the Gibbs potential in the form

$$
Z\left(T, p, N, x_{1}, x_{2}, \ldots, x_{n}\right)=Z_{0}(T, p, N)+\sum_{i=1}^{n} a_{i} x_{i}+\frac{1}{2} \sum_{i, j=1}^{n} b_{i j} x_{i} x_{j}
$$

as a generalization of the Gibbs potential (4) after Taylor expansion with respect to the order parameters $x_{i}(i=1,2, \ldots, n)$. The parameters $a_{i}, b_{i j}=b_{j i}(i, j=$ $1,2, \ldots, n)$ have similar meaning as in the formula (4). Using the orthogonal transformation

$$
x_{i}=\sum_{j=1}^{n} \alpha_{i j} \bar{x}_{j}
$$

the bilinear form in (N.1) can always be diagonalized $\left(b_{i j}=b_{j i}\right)$ and instead of the formulae (N.1) we obtain the following expression (similar to (4))

$$
Z\left(T, p, N, \bar{x}_{1}, \bar{x}_{2}, \ldots, \bar{x}_{n}\right)=Z_{0}(T, p, N)+\sum_{i=1}^{n} \bar{a}_{i} \bar{x}_{i}+\frac{1}{2} \sum_{i=1}^{n} \bar{b}_{i} \bar{x}_{i}^{2}
$$

where $\bar{a}_{i}=\sum_{j=1}^{n} a_{j} \alpha_{j i}$ and $\bar{b}_{i}(i=1,2, \ldots, n)$ are the eigenvalues of the matrix $b_{i j}$. The quantities $\bar{x}_{i}(i=1,2, \ldots, n)$ can be treated as "new" order parameters because of the reciprocal relation

$$
\bar{x}_{i}=\sum_{j=1}^{n} x_{j} \alpha_{j i}
$$


and due to the fact (see (N.4)) that $\bar{x}_{i}(i=1, \ldots, n)$ vanish at the same critical temperature $T_{s}$ as the order parameters $x_{i}(i=1, \ldots, n)$. From the minimum principle $\left(\frac{\partial Z}{\partial \bar{x}_{i}}=0, i=1, \ldots, n\right)$ we obtain

$$
\bar{x}_{i}=-\frac{\bar{a}_{i}}{\bar{b}_{i}}
$$

and this equation can be used to eliminate $\bar{b}_{i}(i=1, \ldots, n)$ from the expression (N.3). We obtain in this way very similar expression to (9), it is

$$
Z\left(T, p, N, \bar{x}_{1}, \bar{x}_{2}, \ldots, \bar{x}_{n}\right)=Z_{0}(T, p, N)+\frac{1}{2} \sum_{i=1}^{n} \bar{a}_{i} \bar{x}_{i}^{2} .
$$

To each $\bar{a}_{i}$ and $\bar{x}_{i}(i=1, \ldots, n)$ we apply analogous assumptions to $(7)$ and (8) where the lower index $i$ should be added. Repeating the calculations similar to the described above (see (10)-(28)) we obtain analogous expressions to (16), (22) and (25)-(28). These new expressions, however, can easily be obtained from (16), (22) and (25)-(28) when replace $b\left[\Delta\left(\frac{\partial x}{\partial T}\right)_{p, N, T=T_{s}}\right]^{2}$ on the rhs of

them by a new quantity $\sum_{i=1}^{n} \bar{b}_{i}\left[\Delta\left(\frac{\partial \bar{x}_{i}}{\partial T}\right)_{p, N, T=T_{s}}\right]^{2}$. This new quantity is irrelevant when forming the quotients (see (29)-(33)) because it cancels out. Thus, the formulae (29)-(33) and the resulting cross relations (34)-(36) remain unchanged. Exactly the same can be said about the formulae (46)-(53) which remain also the same when apply the procedure described in this section with respect to the Helmholtz potential $\bar{Z}=\bar{Z}\left(T, V, N, x_{1}, x_{2}, \ldots, x_{n}\right)$ as a generalization of (39) for the case of superconducting systems with many order parameters.

\section{References}

[1] S.Robaszkiewicz, R.Micnas and K.A.Chao, Phys. Rev. B 26, 3915 (1982).

[2] M.J.Rice, and Y.R.Wang, Phys. Rev. B 37, 5893 (1988).

[3] D. van der Marel, Physica C 165, 35 (1990).

[4] D. van der Marel, in Electronic Properties of High-T $T_{c}$ Superconductors and Related Compounds, edited by J.Fink, Springer-Verlag, Berlin 1990, p.401.

[5] D.I.Khomskii and F.V.Kusmartsev, in Physics and Material Sciences of High Temperature Supercoductors, edited by R.Kossowsky, B.Raveau, D.Wohlleben and S.K.Patapis, Kluwer Academic, Dordrecht 1992.

[6] D. van der Marel and G.Rietveld, Phys. Rev. Lett. 69, 2575 (1992).

[7] G.Rietveld, N.Y.Chen and D. van der Marel, Phys. Rev. Lett. 69, 2578 (1992).

[8] J.Czerwonko, Acta Phys. Polon. B 29, 3885 (1998). 
[9] V.I.Nizhankovskii, Eur. Phys. J. B 18, 397 (2000).

[10] V.I.Nizhankovskii and S.G.Zybtsev, Phys. Rev. B 50, 1111 (1994).

[11] Kh.Eid, E.Gosławska and M.Matlak, Mol. Phys. Rep. 24, 190 (1999).

[12] M.Matlak and M.Pietruszka, Physica C 311, 151 (1999).

[13] M.Matlak and M.Pietruszka, J. Alloys Comp. 291, 21 (1999).

[14] M.Pietruszka and M.Matlak, Acta Phys. Pol. A 96, 275 (1999).

[15] E.Gosławska and M.Matlak, Phys. Stat. Sol. (b) 217, 939 (2000).

[16] M.Matlak and M.Pietruszka, Acta Phys. Pol. A 97, 253 (2000).

[17] M.Matlak, M.Pietruszka, E.Gosławska, B.Grabiec and Kh.Eid, Phase Transitions $\mathbf{7 1}, 73$ (2000).

[18] M.Matlak and M.Pietruszka, Physica B 291, 12 (2000).

[19] M.Matlak, E.Gosławska, B.Grabiec and Kh.Eid, Acta Phys. Pol. A 97, 365 (2000).

[20] E.Gosławska, M.Matlak and Kh.Eid, Acta Phys. Pol. A 97, 713 (2000).

[21] M.Matlak and M.Pietruszka, Sol. St. Commum. 117, 413 (2001).

[22] M.Matlak, M.Pietruszka and R.Rówinski, Phys. Rev. B 63, 52101 (2001).

[23] M.Matlak, M.Pietruszka and R.Rówinski, Phys. Stat. Sol. (a) 134, 335 (2001).

[24] L.D.Landau, Phys. Z. Sovjet. 11, 545 (1937).

[25] W.L.Ginzburg, L.D.Landau, Sovjet Phys. - JETP 20, 1064 (1950).

[26] R.Micnas, J.Ranninger and S. Robaszkiewicz, Rev. Mod. Phys. 62, 113 (1990).

[27] R.Jelitto, J. Phys. Chem. Solids 30, 609 (1969).

[28] K.Elk and W.Gasser, Die Methode der Greenschen Funktionen in der Festkörperphysik, Akademic-Verlag, Berlin 1979. 